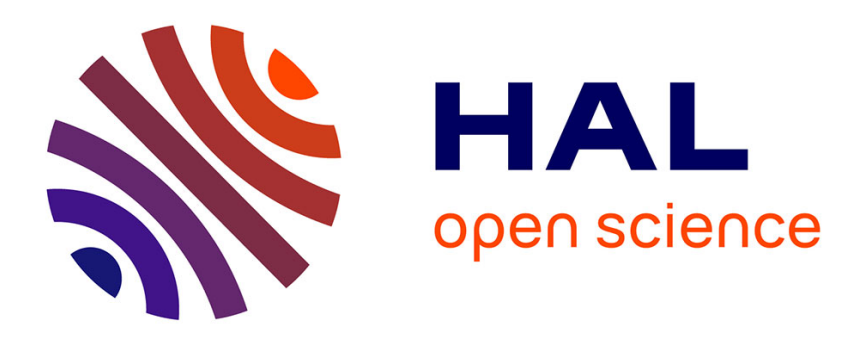

\title{
A Path Dependent Model for Ductile Fracture
}

\author{
B. Goldthorpe
}

\section{To cite this version:}

B. Goldthorpe. A Path Dependent Model for Ductile Fracture. Journal de Physique IV Proceedings, 1997, 07 (C3), pp.C3-705-C3-710. 10.1051/jp4:19973120 . jpa-00255406

\section{HAL Id: jpa-00255406 https://hal.science/jpa-00255406}

Submitted on 1 Jan 1997

HAL is a multi-disciplinary open access archive for the deposit and dissemination of scientific research documents, whether they are published or not. The documents may come from teaching and research institutions in France or abroad, or from public or private research centers.
L'archive ouverte pluridisciplinaire HAL, est destinée au dépôt et à la diffusion de documents scientifiques de niveau recherche, publiés ou non, émanant des établissements d'enseignement et de recherche français ou étrangers, des laboratoires publics ou privés. 


\title{
A Path Dependent Model for Ductile Fracture
}

\author{
B.D. Goldthorpe \\ Defence Evaluation and Research Agency, Fort Halstead, Sevenoaks, Kent, TN14 7BP, U.K.
}

\begin{abstract}
A path dependent model for ductile fracture has been developed which uses the principle of accumulation of damage up to some critical value at which failure occurs. The damage is calculated from void growth equations which depend on the strain path and the value of hydrostatic tension along this path. The model developed on this basis enables the critical damage for failure to be calculated from a simple test such as the tensile test in which the hydrostatic tension can be calculated at all points along the strain path. The method has been verified using some of Bridgman's data over a wide range of strain and hydrostatic tension and more recent data on a high purity iron.
\end{abstract}

\section{INTRODUCTION}

Because of its technological and commercial importance ductile fracture has been the subject of quite intensive and extensive research over a long period. The approaches adopted to study this problem have ranged from the empirical methods of Hancock and McKenzie [1], analysis of void growth such as those by Rice and Tracey [2] and Budiansky et al [3] and the incorporation of void growth models into failure algorithms such as the work of Tvergaard and Needleman [4]. More recently Johnson and Cook [5] have developed an empirical model which sums, strain increments to the fracture strain where the calculation of failure strain is based on void growth models

Progress towards the understanding of ductile fracture as a result of the above work has been substantial and has identified void growth as the damage mechanism, hydrostatic tension as the driving force for void growth and a strain path dependence to damage accumulation. In spite of this progress the development of reliable prediction techniques for ductile fracture has been particularly elusive.

The present work is an attempt to make use of this improved understanding of the problem, particularly the analysis of void growth, and compare with a general theory of path dependence to develop failure models. These methods lead to the identification of a damage path over which the damage accumulation can be calculated up to a critical damage and this can be determined experimentally. In order to integrate over the damage path it is essential to know the hydrostatic tension accurately at all points along the path

The experimental data used to test this approach are from the well-known tensile tests under hydrostatic pressure published by Bridgman [6]. Some of these tests were stopped before failure and then retested at atmospheric pressure. By this means Bridgman was able to subject the materials used to an extremely varied and often severe damage path. In addition the tests are well documented and they therefore provide the best basis available to test a ductile fracture model. Bridgman also, of course, derived equations for the calculation of hydrostatic tension but it has been suggested e.g. Needleman [7] that these equations are inaccurate. Recent work by Goldthorpe and Church [8] has provided a solution to the general axi-symmetric equilibrium equations specific to the tension problem which has shown that Bridgman's equations are in substantial error particularly at large constraints. The accuracy of this new solution has been confirmed by very precise computer modelling of the problem. The use of these equations to calculate hydrostatic tension is of particular significance since they permit some valuable new conclusions to be drawn from Bridgman's pioneering work which were not previously possible.

\section{DEVELOPMENT OF A PATH DEPENDENT MODEL}

The basis of a model with which to calculate damage up to final failure is the description of void growth throughout the process. In line with previous authors the progress of void growth will be identified with the hydrostatic stress which will in general vary continuously along any strain path. Before dealing with this problem we will consider an idealisation in which the measure of void growth D varies under a 
constant hydrostatic stress. Here we can treat $\mathrm{D}$ as the damage which will be a state variable. The progress of damage is shown schematically in figure 1 for two levels of hydrostatic stress. If the path is initially along curve 1 and then the stress is instantaneously changed to path 2 there obviously will be no sudden change in the damage but the rate of damage will change to that of path 2 .

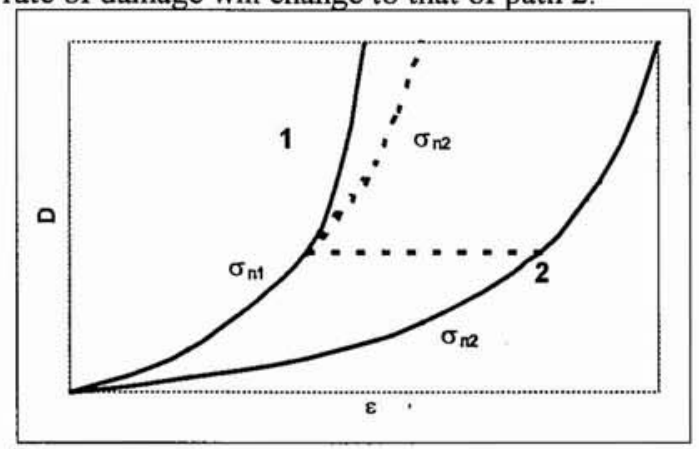

Figure 1; Schematic of Progressive Damage

This behaviour is almost identical to that of path dependent deformation in copper for example. The problem highlighted by figure 1 is that the use of an irreversible variable such as strain precludes the use of an explicit function to describe a path of changing $\sigma_{n}$. In general, therefore, the damage should be described by an equation of the form

$$
\frac{d D}{d \varepsilon}=f\left(D, \sigma_{n}\right)
$$

where $\sigma_{\mathrm{n}}$ is the ratio of the hydrostatic stress to the flow stress. This is usually defined for axial symmetry as

$$
\sigma_{n}=\frac{1}{3}\left(1+\frac{3 \sigma_{r}}{\bar{\sigma}}\right)
$$

For a detailed theory $\mathrm{D}$ would be expected to describe the evolution of damage including the changing void shape and $\sigma_{\mathrm{n}}$ would describe the associated hydrostatic tension.

However for reasons which will soon be apparent a simplified form of (1) will be considered.

$$
\frac{d D}{d \varepsilon}=\phi D
$$

where $\phi$ may be a function of $\sigma_{\mathrm{n}}$ and void shape. This can be integrated at constant $\phi$ taking $\varepsilon=0$ when $D=D_{0}$ to give

$$
D=D_{0} e^{\phi \varepsilon}
$$

More generally on a strain path along which $\phi$ can be evaluated integration gives

$$
D=D_{0} \int_{0}^{\varepsilon} \phi d \varepsilon
$$

We now consider the equation due to Rice and Tracey [2] for the growth of spherical voids written in strain increment form

$$
\frac{1}{V} \frac{d V}{d \varepsilon}=0.85 e^{1.5 \sigma_{n}}
$$

where $V$ is the void volume. Integrating (4) at constant $\sigma_{\mathrm{n}}$ gives

$$
\ln V=0.85 e^{1.5 \sigma_{n}} \varepsilon+k
$$

When $\varepsilon=0 V=V_{0}$, the initial void volume. Thus

$$
\frac{V}{V_{0}}=e^{\eta \varepsilon}
$$

where $\eta=0.85 e^{1.5 \sigma_{n}}$

Thus if we identify the ratio of final to initial void volume as the damage then equations (3) and (5) are equivalent for constant $\sigma_{\mathrm{n}}$ if

$$
\phi=\eta=0.85 e^{1.5 \sigma_{n}}
$$

Equation (5), therefore, provides a simple description of damage accumulation. It does, however, involve a number of simplifying assumptions the most serious of which is that equation (4) is only applicable to spherical voids. Budiansky et al [3] have considered a number of void shapes e.g. cylinders and prolate and oblate spheroids. They showed that if $r$ is the radius of the void then $(I / r)(d r / d t)$ is 
constant for constant $\sigma_{\mathrm{n}}$. Furthermore they showed that this is true for all axi-symmetric voids and the value of the constant is essentially independent of void shape. Clearly if $(1 / r(d r / d t)$ is constant then so is $\left(1 / r^{2}\right)\left(d\left(r^{2}\right) / d t\right)$. Thus if we only consider the damage in a plane normal to the maximum stress we can use equation (4) to describe this damage. Writing $A=\pi r^{2}$ equation (4) gives

$$
\frac{1}{A} \frac{d A}{d \varepsilon}=0.57 e^{1.5 \sigma_{n}}
$$

where we can now treat $A / A_{0}$ as a measure of the damage. It is, therefore, a state variable which is at least approximately independent of changes in void shape and can be written in the form

$$
\frac{d D}{d \varepsilon}=0.57 D e^{1.5 \sigma_{n}}
$$

Equation (6) is now a more specific form of equation (1) and can be integrated along any strain path providing $\sigma_{\mathrm{n}}$ values are known along that path.

At this point it seems appropriate to choose a more convenient, and in many ways, physically more acceptable, definition of damage. If we write

$$
S=\ln \frac{A}{A_{0}}=\ln D
$$

then $S=0$ when $A=A_{0}$ and (7) will now be used as the definition of damage. Equation (6) now gives

$$
\frac{d S}{d \varepsilon}=0.57 e^{1.5 \sigma_{n}}
$$

and integration can be carried out along any strain path to give

$$
S=\int_{0}^{\varepsilon} 0.85 e^{1.5 \sigma_{n}} d \varepsilon
$$

Equation (9) is, therefore, the equation that describes damage accumulation up to some critical value $S_{c}$ at which failure occurs. It is now assumed that $S_{c}$ is a constant for a given material. Once this is done then a simple test, such as a tensile test in which the hydrostatic tension is monitored throughout the test, can be used to evaluate $S_{c}$ using equation (9). Equation (9) can then be used to calculate damage accumulated under other loading conditions and geometries and the strain to failure will be given when $S=S_{c}$.

Before exploring the validity of the above theory and assumptions two improvements can be made to equation (9) which extend its range of validity.

The first of these deals with the fact that Rice's equation is only valid at $\sigma_{n}$ values above about 0.3 . Some combined analytical/numerical results due to Budiansky et al [3] give numerical results down to $\sigma_{n}=0$. These have been used to provide an empirical correction to equation (4) to give

$$
\frac{1}{A} \frac{d A}{d \varepsilon}=0.67 e^{3 / 2 / \sigma_{n}-0.04 \sigma_{n}^{-1.5}}
$$

The second correction deals with the problem of very large values of $\sigma_{n}$ since it is clear form equation (9) that at these large values the strain falls to extremely low values which may be much less than the elastic strain. The reason for this is that plasticity theory always takes the sum of three principal strains to be zero and therefore under a hydrostatic tension the strain must always be zero. However, if we consider a cube containing a void subject to such stresses the increase in void area divided by the cube area will give the minimum possible strain. Thus if there are $N$ defects of initial area $A_{\diamond}$ per unit area then since

The strain due to void growth is then

$$
\begin{gathered}
S=\ln \frac{A}{A_{0}} \\
A=A_{0} e^{S}
\end{gathered}
$$

$$
\varepsilon_{V}=N A_{0}\left(e^{s}-1\right)
$$

and this should be added to calculated strain values although it will normally be small compared to the distortional strains.

The final equation for damage accumulation is therefore

$$
S=0.67 \int_{0}^{\varepsilon_{a}} \exp \left[1.5 \sigma_{n}-0.04 \sigma_{n}^{-1.5}\right] d \varepsilon
$$

Total strain $\varepsilon_{\mathrm{t}}=\varepsilon_{\mathrm{a}}+\varepsilon_{\mathrm{v}}$ 


\section{DAMAGE ACCUMULATION IN TENSION}

\subsection{Calculation of Hydrostatic Tension}

Bridgman's equations for hydrostatic tension in deforming rods have been found to be inaccurate $[7,8]$. The principal reason for this is that Bridgman only solved the equation for radial equilibrium. Recently Goldthorpe and Church [8] have solved the second order equation

$$
\frac{\partial^{2} \sigma_{r}}{\partial^{2}}+\frac{1}{r} \frac{\partial \sigma_{r}}{\partial}=\frac{\partial^{2} \sigma_{z}}{\partial z^{2}}
$$

specifically for the tension problem. Equation (13) gives the combined axial and radial equilibrium in a neck on the assumption that $\sigma_{\mathrm{r}}=\sigma_{\theta}$. The solution for the radial stress is

$$
\sigma_{r}=\sigma_{c} \operatorname{Cosnz}\left[1-\frac{n^{2} r^{2}}{2^{2}}+\frac{n^{4} r^{4}}{2^{2} \cdot 4^{2}}-\ldots .\right]
$$

where $\sigma_{\mathrm{c}}$ is the stress on the axis and $\mathrm{n}$ is a Bessel coefficient and $z=0$ at the plane of symmetry in the neck For a rigid plastic solid and Tresca yield criterion

$$
\sigma_{c}=0.802 \frac{a}{\rho}
$$

where $a$ is the current radius and $\rho$ is the radius of curvature. The value of $n$ is 2.4 and the coefficient in (15) is calculated from $\mathrm{n}$ and the boundary conditions.

One of the features of the solution (14) is that the value of $n$ and $\sigma_{c}$ depend on the material model through the variation of $\sigma_{z}$ with $z$. The effect of this is to reduce the constraint necessary to maintain equilibrium since strain hardening and strain rate effects provide some of the additional strength that is required. This is not a major problem in normal tensile tests for most materials since the strain hardening rate has reduced considerably by the time the neck forms. It is more of a problem with machined notches since these can be used in annealed material where the strain hardening rate can be initially very high. It is. therefore, important in studying the effect of hydrostatic tension on fracture that both the material model and the geometry are used to calculate constraint. A common observation with severe notches is that the notch radius of curvature increases with strain which is clear evidence that strain hardening rather than hydrostatic tension is dominating the stress distribution.

\subsection{Analysis of Bridgman's Tensile Tests}

Among the many tests carried out by Bridgman some were of particular significance to the ductile fracture problem. These were tests which were started under conditions of large hydrostatic pressure and then stopped at various stages. This provided specimens with necks of varying severity which, unlike machined notches, were in equilibrium in the fully plastic state. Retesting at atmospheric pressure provided large hydrostatic tension and small strain to failure.

The test data we shall use here is the standard atmospheric pressure test in which a/ $\rho$ was monitored for a baseline calculation of the critical damage factor $S_{c}$. In addition test data will be used where prestraining took place at high pressure but was stopped before $\sigma_{n}$ (as calculated by equation (15)) exceeded zero while under pressure. It is assumed therefore that in these tests void growth was negligible before the atmospheric pressure part of the test. One test will also be included where $\sigma_{n}$ reached a significant positive value while under pressure.

The most complete set of data is on what Bridgman classified as tempered pearlite. The analysis of the ambient test using equations (15) and (12) gave an $S_{c}$ value of 1.87. Figure 2 shows the damage as a function of both strain and $\sigma_{\mathrm{n}}$ for this test where it can be seen that $\sigma_{\mathrm{n}}$ is constant until necking starts as would be expected. It then increases fairly sharply but from then on the rate of increase progressively decreases. The damage $S$ increases monotonically and at a steadily increasing rate.

The tests which were started at high pressure and then continued at atmospheric pressure required a more detailed analysis. The progress of $\sigma_{n}$ at high pressure had to be calculated first and then the progress of $\sigma_{n}$ calculated when the test was continued at atmospheric pressure. When $\sigma_{n}$ was positive this was included in the calculation of $S$ and also in the eventual failure damage $S_{c}$. All of the essential data from these tests is included in Table I. 


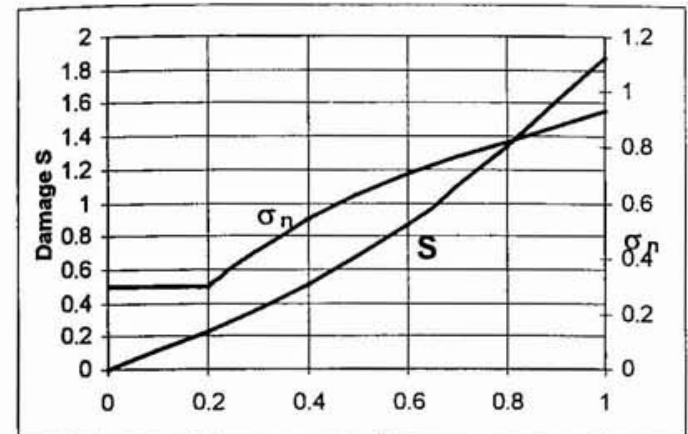

Figure 2; Damage and $\sigma_{\mathrm{n}}$ paths at atmospheric pressure

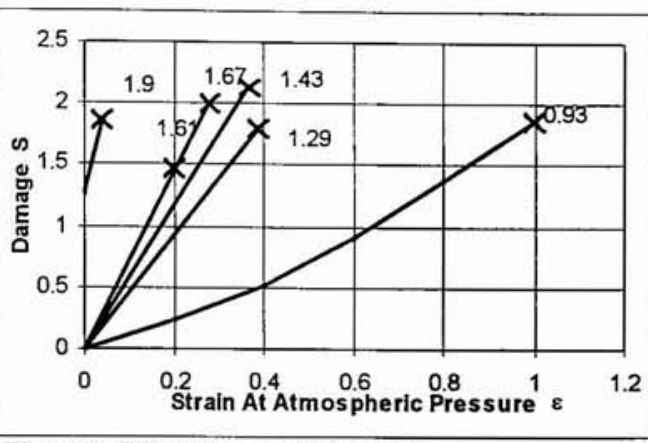

Figure 3; Effect of $\sigma_{\mathrm{n}}$ on strain path

\begin{tabular}{|cccccc|}
\hline $\begin{array}{c}\text { Initial test } \\
\text { pressure } \\
\text { GPa }\end{array}$ & $\varepsilon$ at pressure & $\begin{array}{c}\text { Final } \sigma_{n} \text { at } \\
\text { pressure }\end{array}$ & $\begin{array}{c}\text { Range of } \sigma_{n} \\
\text { at } \\
\text { atmospheric } \\
\text { pressure }\end{array}$ & $\begin{array}{c}\varepsilon \text { at } \\
\text { atmospheric } \\
\text { pressure }\end{array}$ & $S_{c}$ \\
\hline & & & $0.33-0.93$ & 1.03 & 1.86 \\
2.00 & 1.15 & -0.63 & $0.97-1.29$ & 0.45 & 1.84 \\
2.52 & 2.19 & -0.016 & $1.51-1.67$ & 0.28 & 2.02 \\
2.45 & 1.73 & -0.21 & $1.30-1.43$ & 0.38 & 2.16 \\
2.52 & 2.15 & -0.017 & $1.51-1.61$ & 0.20 & 1.47 \\
2.54 & 3.29 & 0.3 & $1.88-1.90$ & 0.05 & 1.92 \\
\hline
\end{tabular}

Table I

These tests show quite a wide range in all of the important variables of range of $\sigma_{n}$, final $\sigma_{n}$ and strain to failure. However the calculated damage $S_{c}$ shows good consistency with the exception of the value at 1.47. An examination of Bridgman's data shows this particular test is generally out of line with the other tests suggesting a not unexpected influence of segregation of inclusions which has a large effect on the small testpieces used. Even when this test is included the level of agreement is sufficient to provisionally accept the damage $S_{c}$ as a material characteristic which describes the ductile fracture resistance. These results are summarised in figure 3 where the damage path for all the tests is shown. This brings out more clearly the consistency of $S_{c}$ in that it is independent of both the path and the final value of $\sigma_{n}$. Of particular interest is the test which attained a damage value of 1.34 under high pressure followed by additional damage of 0.58 at atmospheric pressure to give a total of 1.92 . This is clearly in agreement with all other tests in which the entire damage was sustained at atmospheric pressure. Clearly this data could also be presented as a $\sigma_{\mathrm{n}} / \varepsilon$ plot in common with other authors [1] but with a greater range of $\sigma_{\mathrm{n}}$.

\section{DISCUSSION}

Since much of the previous work on ductile fracture is based on void growth they make use of the equation due to Rice and Tracey [2]. It is not surprising, therefore, that other models for fracture e.g. [1,5] are similar to equation (9) although none of these authors used a path integral approach or any other form of integral. Hancock and Mackenzie [1] used a constant $\sigma_{n}$ although $\sigma_{n}$ could not have been constant in their experiments and in the light of present knowledge the calculation of $\sigma_{n}$ was inaccurate. Johnson and Cook [5] expressed the fracture strain as a function of $\sigma_{n}$ and various constants in an empirical equation. A summation process was then used to calculate progressive damage. Some of the problems associated with this technique arise from the expression of strain as a function of other variables in a path dependent process. Also any additive constants in the fracture strain equation, which must include volumetric strain and strain to nucleation, are not included in the accumulation process which depends only on distortional strains and this leads to inconsistencies particularly at large $\sigma_{\mathrm{n}}$

The present method has attempted to overcome these problems by defining damage as the volumetric strain and calculating the damage along a distortional strain path along which $\sigma_{n}$ is known. The successful application of this technique to Bridgman's data in the present work can be primarily attributed to two main causes. These are the intrinsic validity of the void growth models $[2,3]$ and the accurate calculation of $\sigma_{n}$ [8]. If Bridgman's equations had been used for the calculation of $\sigma_{n}$ then no agreement would have been obtained, in fact the results look quite random. The difference between Bridgman's analysis and that due to Goldthorpe and Church [8] is very large at large constraint. At a relative radius of curvature of 2 the value of $\sigma_{\mathrm{r}}$ on the axis differs by a factor of $2.3[8]$ giving the higher result. 
It would appear, therefore, that the importance of $\sigma_{n}$ being accurately determined may have prevented good agreement being obtained in other work. The probable reason for this is that the test techniques normally used involve machined notches or necks. If the constraint is calculated by a method which applies to the fully plastic state the results will be in substantial error. If the material is strongly strain hardening the results will be in even greater error since the strain hardening gradient along the axis takes the place of constraint.

The importance of this is brought out in some results by Mirza [9] on a particularly ductile form of iron. These results are shown in figure 4 which shows strain against $\sigma_{n}$ for different notch geometries. Curve 1 shows the calculation of $\sigma_{n}$ and strain on the centreline elements using the Lagrangian hydrocode DYNA 2D and an accurate material model for the iron. Thus the conditions along the axis are radically different to those estimated from standard methods for notch geometry. A tensile test of the same material that was tested by Mirza was used to determine the damage factor $S_{c}$ by means of equation (12) and this gave a value of 5.4. This value was used to calculate the $\sigma_{n} / \varepsilon$ curve shown as Curve 2 in figure 4 . Clearly the agreement between the hydrocode calculation and the methods of this paper is excellent which gives added support to the importance of accurate calculations of $\sigma_{n}$.

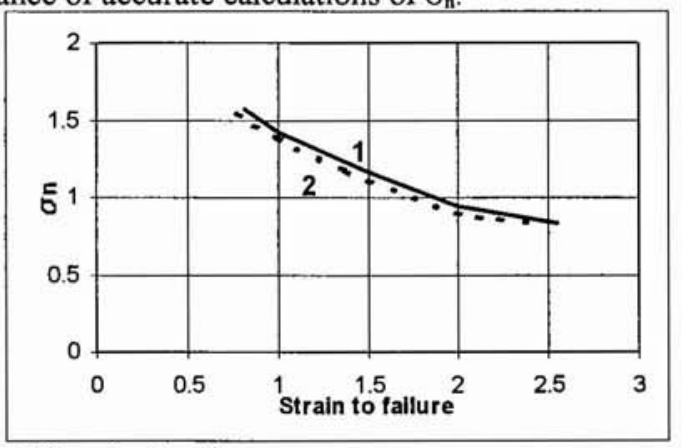

Figure 4; Effect of $\sigma_{n}$ on Strain to Failure for Pure Iron

The general level of agreement between experimental fracture data and the methods of fracture prediction developed in this paper has proved extremely encouraging over the range of data that is available for comparison. Within this range it appears to provide a predictive method which is ideally suited to computer modelling methods since equation [12] is easily evaluated by these methods for materials where the deformation algorithms and $S_{c}$ values are known. Clearly extension of the method to a much wider range of $\sigma_{\mathrm{n}}$ values will depend on the extension of fracture experiments to these conditions.

\section{REFERENCES}

[1] Hancock JN and McKenzie AC; Jnl Mech \& Phys Solids, 24, 1976, 147

[2] Rice JR and Tracey DM; Jnl Mech \& Phys Solids, 17, 1969, 201

[3] Budiansky B, Hutchinson JN and Slutsky S; Mechanics of Solids, Pergamon, 1980, 13

[4] Tvergaard V and Needleman A; Acta Met, 32, 1984, 157

[5] Johnson GR and Cook WH; Engineering Fracture Mechanics, 21, 1985, 31

[6] Bridgman PW; Large Plastic Flow and Fracture, McGraw-Hill, 1952

[7] Needleman A; Jnl Mech \& Phys Solids, 20, 1972, 111

[8] Goldthorpe BD and Church P; to be published 1997

[9] Mirza MS; Deformation and Fracture of Ductile Metals at High Strain Rates, phD Thesis, University of Leeds, 1993 